EPJ manuscript No.

(will be inserted by the editor)

\title{
Dynamic Soft Elasticity in Monodomain Nematic Elastomers
}

\author{
A. Hotta and E.M. Terentjev \\ Cavendish Laboratory, University of Cambridge, Madingley Road, Cambridge CB3 0HE, U.K.
}

October 22, 2018

\begin{abstract}
We study the linear dynamic mechanical response of monodomain nematic liquid crystalline elastomers under shear in the geometry that allows the director rotation. The aspects of time-temperature superposition are discussed at some length and Master Curves are obtained between the glassy state and the nematic transition temperature $T_{n i}$. However, the time-temperature superposition did not work through the clearing point $T_{n i}$, due to change from the "soft-elasticity" nematic regime to the ordinary isotropic rubber response. We focus on the low-frequency region of the Master Curves and establish the power-law dependence of the modulus $G^{\prime} \propto \omega^{a}$. This law agrees very well with the results of static stress relaxation, where each relaxation curve obeys the analogous power law $G^{\prime} \propto t^{-a}$ in the corresponding region of long times and temperatures.
\end{abstract}

PACS. 83.80.Dr Elastomeric polymers - 61.30.-v Liquid crystals. - 83.10.Nn Polymer dynamics

\section{Introduction}

For the last two decades, thermotropic liquid crystalline polymers (LCP) have been a subject of increasing activity due to their fascinating potential in technical applications, ' as well as some fundamentally different properties in comparison to ordinary liquid crystals. Typical LCP consist of a polymer backbone and mesogenic groups. The backbone is usually made of siloxane or acrylic chains and the mesogenic groups are either grafted (end-on or side-on) using a flexible spacer, thus making a side-chain LCP, or incorporated directly into the polymer backbone (mainchain LCP). When the polymer is chemically crosslinked, it forms a three-dimensional permanent network connected with mesogenic moieties in what is called Liquid Crystalline Elastomers (LCE) [1], or gels if the network is swollen by a solvent. Many new physical results have been reported for LCE, but perhaps the most important novelty, unique for nematic LCE materials, is the effect of soft elasticity, where imposed strains could be completely accommodated without any (or with very low) elastic response due to the relaxation of internal microstructure. These unusual mechanical properties of LCE can be directly applied in technology, using LCE as anomalous damping materials, as well as artificial muscles or mechanical transducers.

The early research of LCP rheology, focused mostly on the viscosity of polymers, was summarized in [2]. Many interesting results have come to light once the mesogenic groups were incorporated into linear or branched polymer systems. The classical Leslie-Ericksen theory of lowmolecular-weight liquid crystals is, in most of the cases, not applicable to LCP systems because of the underlying polymer dynamics making the response distinctly non-
Newtonian. Hanus et al. [3] measured dynamic-mechanical properties of a weakly crosslinked main-chain LCP having crystalline, smectic B, smectic A and isotropic phases. They only observed a clear step in the linear shear modulus $G^{\prime}$ at the transition temperature from smectic A to the isotropic state. In the study of LCP having smectic $\mathrm{C}$, smectic A, nematic and isotropic phases [4], Pakula reported that decreases in the linear modulus were detected at the smectic/nematic transition as well as the glass/smectic transition. Also interestingly, although it was not discussed theoretically at that time, the increase of the modulus could be seen during the transition from the nematic to the isotropic phase. The issues of timetemperature superposition in LCP rheology have been actively discussed by several groups such as Colby et al. [5, Gallani et al. [7, 8], Fourmaux-Demange et al. [6] and Weilepp et al. [9]. All of these works also reported the characteristic power-law frequency dependence of the storage modulus $G^{\prime}(\omega)$. Colby et al. used a sample possessing three phases (isotropic, nematic and smectic A) and concluded that the viscoelastic response is very sensitive to smectic-nematic and smectic-isotropic transitions, but insensitive to the nematic-isotropic transition. The timetemperature superposition worked only at the nematicisotropic transition. Approaching the glass transition, $G^{\prime}(\omega)$ obeys a power law of $G^{\prime} \propto \omega^{0.75}$. The authors suggested that this power law might be connected to the large polydispersity of their sample [5], which was also argued by Fourmaux-Demange et al. [6] for their different LCP system. Gallani et al. measured dynamic-mechanical response of several LCP materials and also found a power law behaviour of the storage modulus $G^{\prime}$, with the exponent of $0.44,0.6,0.67$ or 0.75 depending on the sample. Here 
A. Hotta, E.M. Terentjev: Dynamic Soft Elasticity in Monodomain Nematic Elastomers

again, the time-temperature superposition worked only during the nematic-isotropic transitions. This was attributed [6, 8 to the transient elastic clusters of macroscopic size (a few tens of micrometers), floating in a viscous medium. One may recall that a classical Rouse dynamics of polymer chains would lead to a square-root dependence $G^{\prime} \propto \omega^{0.5}$ [10].

In this paper we shall find that the power law dependence is also effective in crosslinked LCE materials. In their parallel study of LCE, Gallani et al. and Weilepp et al. 8,9] also clearly stated that the rheological response did not change significantly when crossing an isotropicnematic phase boundary, whereas there has been a crucial change at the nematic-smectic A transition. The powerlaw frequency dependence of the elastic moduli has again been reported, with $G^{\prime} \propto \omega^{0.3}$ in the smectic A phase. In the isotropic phase, the exponent was found to be 0.5 , similar to the Rouse model prediction. A more detailed recent study of nematic LCE rheology by Stein et al. [11] also reported $G^{\prime} \propto \omega^{0.5}$ in a polydomain nematic elastomers, but could not obtain Master Curves for monodomain, aligned nematic materials. Working with such monodomain nematic rubbers, Clarke et al. [12,13] reported a significant difference in response between polydomain samples (where the nematic director is disordered on the scale above few microns) and monodomain nematic rubbers (with the director is macroscopically aligned along the shear direction, or perpendicular to it). A substantial decrease in the lowfrequency storage modulus $G^{\prime}$ was registered as the temperature was decreasing through nematic-isotropic transition. This unusual effect has been theoretically described by a continuum theory of linear viscoelastic response in oriented monodomain nematic elastomers [14].

Continuous stress relaxation is a related subject, which offers a different angle of approach to the complex problem of polymer dynamics exploring the time, rather than the frequency domain. Chasset and Thirion, in their classical paper [15, described a power law decay in ordinary crosslinked polymer networks such as the isoprene rubber (IR) and the butadiene-styrene copolymer rubber (SBR). They found that, after application of a constant strain, the stress response decreases with time, reaching at long times a characteristic power-law regime $\propto 1 / t^{0.1-0.15}$ at very long times. In the linear regime (at small enough deformations), in an incompressible material (when the extension modulus is simply three times the shear modulus $G$ ), this translates directly into the relaxation of the effective modulus $G(t)$. Many subsequent experiments on stress relaxation, performed by different groups, have supported this long-time power law decay with a small exponent. Theoretical analysis of [16], applying the reptation ideas for a network with long free dangling chain ends, has shown that the retraction of these chains could account for the observed stress relaxation law $\sigma \propto t^{-a}$ with a small exponent $a \sim 0.1-0.15$.

In liquid crystalline networks, the early results shown a different response. The problem in such experiments is always the need to access very long times, when the main transient processes in the polymer network have relaxed and only the slowest mode remains. A peculiar dual regime of long-time relaxation in a polydomain siloxane nematic LCE has been reported by Clarke et al. [17]. A characteristic time $t^{*} \sim 3000 \mathrm{~s}$ separated a regime of fast power-law relaxation, $G \propto t^{-0.5}$ or higher, and a very slow relaxation at the later stage, fitted in [17] with an inverse-logarithmic law $\sigma \sim\left[1+\ln \left(t / t^{*}\right)\right]^{-1}$. An even faster power-law decay was reported by Hotta et al. 18] in their study of acrylic nematic LCE, $G \propto t^{-0.67}$ in the early stage of stress relaxation. At very long times, they find the different slow power law decay with the exponent $\sim 0.15$, similar to the one observed in natural crosslinked rubber by Chasset and Thirion.

From this brief summary, it is clear that the powerlaw dependence of linear response function on time or frequency is a recurrent feature of LCE rheology. On the other hand, it is equally clear that the response is nonuniversal and the particular features of the response depend on the material composition and also on the nematic director texture and orientation with respect to the applied strain. In this paper we describe a simultaneous study of dynamic-mechanical and stress-relaxation responses for the two well-characterised nematic LCE materials. One sample is a "classical" siloxane side-chain nematic rubber first prepared and described in great detail by Finkelmann et al. and others [19, 20, 11, 12]. The other material had a high content of main-chain nematic polymer strands, which makes the effective chain anisotropy very high [21, but also leads to a very slow dynamics. We shall examine in some detail the issues of time-temperature superposition, which is necessary to extrapolate the response function into the regions of time or frequency not practically accessible on experiment. We find that the results of two such different experimental techniques are, in fact, in a good agreement: the observed frequency power laws $G^{\prime} \propto \omega^{a}$ are matched by the corresponding relaxation laws $G \propto t^{-a}$, if the corresponding ranges of time and frequency are examined.

\section{Material Characterisation}

Two nematic elastomers were prepared in our group following the synthetic procedure pioneered by Finkelmann et al. [19]. The polymer backbone was a standard polymethylhydrosiloxane with approximately $60 \mathrm{Si}-\mathrm{H}$ units per chain, obtained from ACROS Chemicals. The pendant mesogenic groups were 4'-methoxy phenyl-4-(1-buteneoxy) benzoate (MBB), as illustrated in Fig. 11, attached to the backbone end-on via the hydrosilation reaction in the presence of a commercial platinum catalyst COD, obtained from Wacker Chemie. The first monodomain nematic elastomer was prepared following the two-step crosslinking reaction 19], with 1,4 di(11-undecene) benzene (11UB), a small flexible difunctional molecule deemed to have relatively minor effect on the overall mesogenic properties of the liquid crystalline polymer (synthesized in the house).

The second network was prepared following the similar procedure, but using di-vinyl terminated polymer chains 


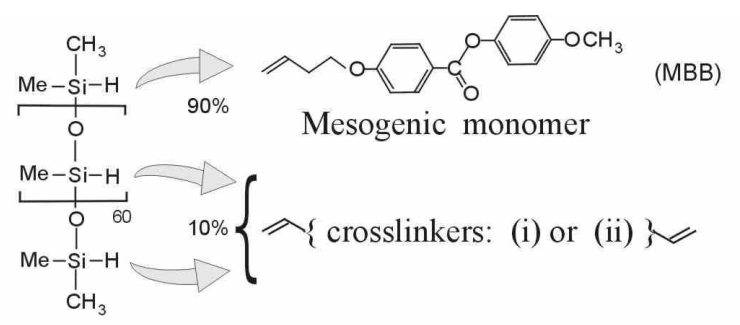

(a)

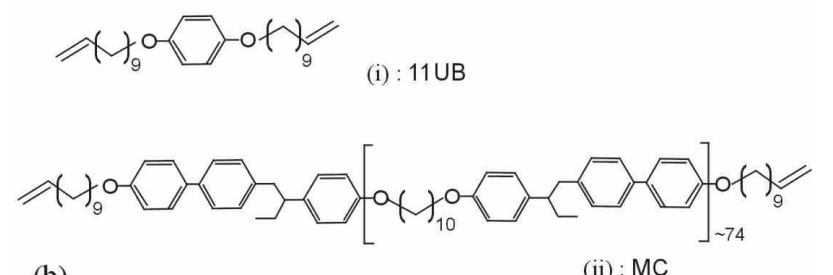

(b)

(ii) : MC

Fig. 1. Schematic illustration of the materials used in this work. (a) Siloxane backbone chain with $\mathrm{Si}-\mathrm{H}$ groups reacting with $90 \mathrm{~mol} \%$ mesogenic phenyl-benzoate side groups, MBB and $10 \mathrm{~mol} \%$ of di-vinyl crosslinking groups: (b) flexible smallmolecule 1,4 alkeneoxybenzene, 11UB (resulting in the $\mathrm{SiH}$ material), and the main-chain nematic polymer of 1-biphenyl-2phenyl butane, MC (giving the SiMC material).

of $\alpha$ - $\{4$-[1-(4'-\{11-undecenyloxy $\}$ biphenyl)-2-phenyl] butyl)$\omega$-(11- undecenyloxy) poly-[1-(4- oxydecamethyleneoxy)biphenyl -2-phenyl] butyl (MC) that themselves make a main-chain LCP [22]. In our case, the crosslinking chains had $\sim 75$ rod-like monomers between the terminal vinyl groups (determined by GPC, polydispersity $\sim 2$ ). As a result, the properties of a nematic LCE are dominated by these long main-chain LCP strands connecting the siloxane side-chain molecules (as the Table 1 indicates, the overall $\mathrm{MC}$ content is $\sim 58 \%$ ). In both cases, the crosslinking density was kept at $10 \mathrm{~mol} \%$ of the reacting bonds in the siloxane backbone, so that on average, each siloxane chain has nine pendant mesogenic groups between crosslinking sites.

Both elastomers were prepared from the same batch of backbone and side-group mesogenic materials, with the same relative concentration of crosslinking groups.

Table 1 summarizes chemical and thermal characteristics of both materials. Equilibrium transition temperatures were determined on a Perkin Elmer Pyris 1 differential scanning calorimeter (DSC), extrapolating to low cooling rates, and the nematic phase identified by polarizing optical microscopy and X-ray scattering. The average degree of chain anisotropy was identified by observing the uniaxial thermal expansion of LCE samples as a function of temperature. Thermal expansion measurements were made by suspending the samples, without load, in a glassfronted oven and measuring the variation in natural length of the samples with temperature, $L(T)$; see 21] for further details.

\begin{tabular}{|l|c|c|c|c|c|c|} 
Sample & $11 \mathrm{UB}$ & $\mathrm{MC}$ & $\mathrm{SC}$ wt & $r$ & $T_{g}$ & $T_{n i}$ \\
\hline $\mathrm{SiH}$ & $10 \%$ & $0 \%$ & $87 \%$ & 1.5 & $3^{\circ} \mathrm{C}$ & $86^{\circ} \mathrm{C}$ \\
$\mathrm{SiMC}$ & $9 \%$ & $1 \%$ & $42 \%$ & 2.8 & $2^{\circ} \mathrm{C}$ & $107^{\circ} \mathrm{C}$ \\
\hline
\end{tabular}

Table 1. Proportions (in mol\%) of crosslinkers 11UB and MC in the overall crosslinking composition (of the fixed total of $10 \%)$, the corresponding volume fraction of side-chain mesogenic groups (in wt\%), the average chain anisotropy $r$ at room temperature, temperatures of glass and nematic-isotropic transitions. The glass transition temperatures are approximate, with an error of at least $\pm 5^{\circ} \mathrm{C}$.

\begin{tabular}{|l|c|c|c|c|} 
& \multicolumn{2}{|c|}{ Sandwich } & \multicolumn{2}{c|}{ Tape } \\
\hline & Thickness & Area & Thickness & Area \\
\hline SiH & $0.25 \mathrm{~mm}$ & $79 \mathrm{~mm}^{2}$ & $2 \mathrm{~mm}$ & $1.9 \mathrm{~mm}^{2}$ \\
& & $\left(\pi \times 5^{2}\right)$ & & $(7.4 \times 0.25)$ \\
SiMC & $0.15 \mathrm{~mm}$ & $36 \mathrm{~mm}^{2}$ & $2 \mathrm{~mm}$ & $1 \mathrm{~mm}^{2}$ \\
& & $\left(\pi \times 3.4^{2}\right)$ & & $(6.7 \times 0.15)$ \\
\hline
\end{tabular}

Table 2. Samples and their dimensions for the simple shear experiment in two mounting geometries, see text.

\section{Dynamic Mechanical Analysis}

In this paper, we used the dynamic mechanical apparatus (DMA) Viscoanaliser VA4000, from Metravib RDS. In the previous work 13, 12] we have already examined the anisotropy of the shear response depending on the orientation of the nematic director, see the sketch in Fig. 2(a). Only the rotation-inducing geometry reflects the effects of soft elasticity and here we concentrate on this particular case. The way in which the samples were mounted in the DMA is shown in Fig. 2(b). It was important to choose the correct mounting for a given temperature range because of the great variation in the magnitude of response between the low-temperature (or high-frequency) glassy regime and the high-temperature rubber plateau. We used two different ways of sample mounting: The more stiff "sandwich" geometry, with each sample having the large area of contact with the clamps and the small thickness, was used to collect weaker signals from softened samples at higher temperature (in our DMA setup the thin-film sample had a round shape). The "tape" mounting geometry, in which the rectangularly cut thin-film sample is clamped sideways with only the small cross-section area, results in smaller forces and allows to measure the modulus of very rigid samples up to the glass plateau. The Table 2 gives the dimensions of typical sample mounting.

A geometry of uniaxial extension, more commonly used in studies of equilibrium stress-strain relaxation in rubbers, is less appropriate for an oscillating regime because of slow relaxation and incomplete sample recovery on each cycle. In the chosen simple shear setup, the middle part of the sample holder is forced to move in an oscillating fashion, maintaining the small shear strain - between 0.003 and 0.004 (that is, $0.3-0.4 \%$ ). This value was chosen from a separate strain-ramping experiment, finding the range of strains that still return a linear response in the whole of the chosen frequency range. 

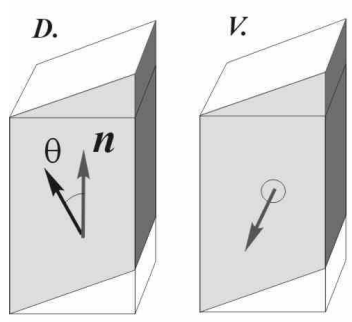

(a)

Fig. 2. (a) The geometry of simple shear with two principal orientations of the initial director $\boldsymbol{n}$, labelled $\boldsymbol{D}$ (for $\boldsymbol{n}$ along the shear displacement) and $\boldsymbol{V}$ (vorticity). No director rotation, hence no soft elasticity, occurs in the $\boldsymbol{V}$-geometry [12]. (b) The symmetric sample mounting in the DMA device, in the $\boldsymbol{D}$-geometry. See text for the sample dimensions used in different setups.

\subsection{Temperature Scans}

The materials were studied over a wide temperature range encompassing the glass and the nematic/isotropic transitions. Typically the temperature scans ranged from $-50^{\circ} \mathrm{C}$ to $130^{\circ} \mathrm{C}$, at a number of fixed frequency values. Figure 3 shows the results for the storage modulus $G^{\prime}$ at just two frequencies, 0.01 and $100 \mathrm{~Hz}$, for the $\mathrm{SiH}$ sample. The storage modulus $G^{\prime}$, which is the real part of the linear complex modulus $G^{*}(\omega)$ [24] and the loss factor $\tan \delta=G^{\prime \prime} / G^{\prime}$ (with $G^{\prime \prime}$ the imaginary part of $G^{*}(\omega)$ the loss modulus) were plotted against the temperature. As we mentioned above, it is technically difficult to obtain a clean mechanical signal from the sample that undergoes a change in stiffness of up to $10^{4} \rightarrow 10^{9} \mathrm{~Pa}$. Therefore, we used the two ways of mounting the samples, the "sandwich" at high and the "tape" at low temperatures. One can see in Fig. 3, as well as in Fig. Af for the SiMC material, that the two portions of the full curve, for the tape and sandwich modes, are connecting quite successfully without any additional adjustment (the data were discarded if the vertical error was reaching $15 \%$, on the assumption of a mounting fault). This indicates good reliability of DMA measurements for both the storage modulus $G^{\prime}$ and loss factor $\tan \delta$.

The glass plateau modulus for both $\mathrm{SiH}$ and SiMC materials reaches up to nearly $10^{9} \mathrm{~Pa}$, which is a reasonable value in polymeric materials. Between $0^{\circ} \mathrm{C}$ and $10^{\circ} \mathrm{C}$, both $\mathrm{SiH}$ and $\mathrm{SiMC}$ undergo the glass/nematic transition, showing a dramatic decrease in $G^{\prime}$ on cooling, and the associated steep rise in $\tan \delta$. The storage modulus decreases down to $\sim 10^{5} \mathrm{~Pa}$ which is an expected order of magnitude in rubbery phases. However, both materials show a substantial drop in $G^{\prime}$ in the nematic phase, below $T_{n i}$. This effect, most pronounced at low frequencies, is the dynamic soft elasticity: the reduction in the modulus and the rise in internal mechanical dissipation caused by the underlying director rotations induced by shear 114, 12. The relative depth of the drop in $G^{\prime}$ is higher in SiMC, the material with a higher chain anisotropy due to its main-chain LCP component. Above $T_{n i}$, the storage modulus returns to its

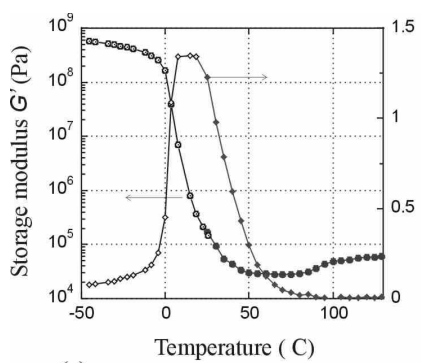

(a)

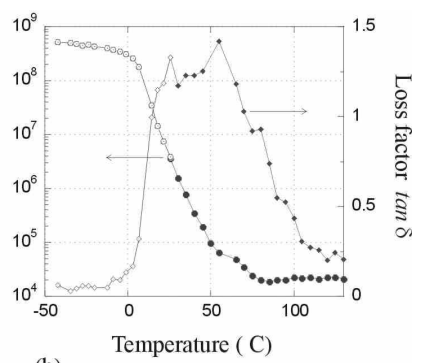

(b)
Fig. 3. Storage modulus $G^{\prime}$ (circles) and loss factor tan $\delta$ (diamonds) as functions of temperature at frequencies of 0.01 (a) and $100 \mathrm{~Hz}$ (b). The graphs combine the data obtained from the tape (open symbols) and the sandwich (filled symbols) sample mounting geometry. Results for $\mathrm{SiH}$.

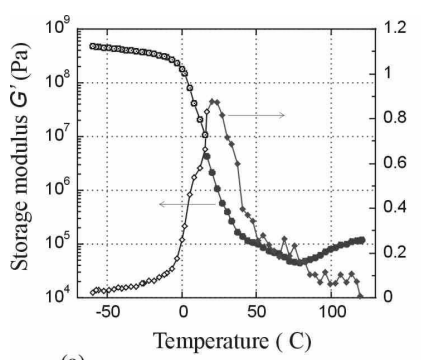

(a)

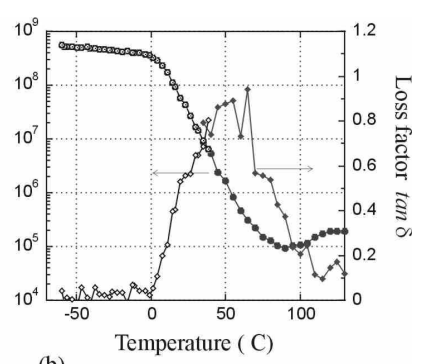

(b)
Fig. 4. Storage modulus $G^{\prime}$ (circles) and loss factor tan $\delta$ (diamonds) as functions of temperature at frequencies of 0.01 (a) and $100 \mathrm{~Hz}$ (b). The graphs combine the data obtained from the tape (open symbols) and the sandwich (filled symbols) sample mounting geometry. Results for SiMC.

(higher) value characteristic of ordinary isotropic rubbers. At very low frequencies $G^{\prime}$ is slightly rising with temperature, revealing that the sample is in a crosslinked rubbery state with its equilibrium shear modulus $G \sim n_{x} k_{B} T$ (where $n_{x}$ is the crosslink density and $k_{B}$ is Boltzmann factor).

\subsection{Frequency Scans}

Figures 5 and 6 show the same data for the storage modulus $G^{\prime}$, but this time plotted against frequency at a set of constant values of temperature. In our experiments, the frequency was changing between 0.01 to $900 \mathrm{~Hz}$, measured 45 points in total. However, we were obliged to discard higher frequencies: the DMA data sets must pass a series of tests on internal consistency and failing these tests indicates one of several possible problems with acquisition (most common is the internal resonance of the mechanical frame). Only the reliable range of results is presented in Figs. 5 and 6 .

The range of storage modulus variation with frequency matches well with the temperature scans, from $10^{9} \mathrm{~Pa}$ down to $10^{4}-10^{5} \mathrm{~Pa}$. As in the temperature scans, the average value of $G^{\prime}$ is rising on heating, when each sample approaches its isotropic rubbery phase and the mesogenic effects weaken. In each of the figures, the plot (b) shows the expanded version of the high-temperature re- 

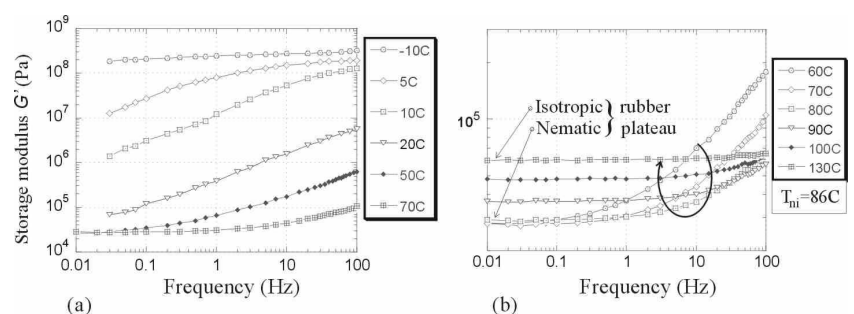

(a)

(b)

Fig. 5. Storage modulus $G^{\prime}$ as a function of frequency at temperatures ranging from $-10^{\circ} \mathrm{C}$ to $130^{\circ} \mathrm{C}$, labelled on plots; results for $\mathrm{SiH}$. Plot (a) shows the set of lower temperatures, including the glass and the nematic rubber plateau regimes and clearly suggesting the time-temperature superposition. Plot (b) shows the higher temperatures, below and above $T_{n i}$, indicating the rise in the rubber plateau value in the isotropic phase: the dynamic signature of soft elasticity.

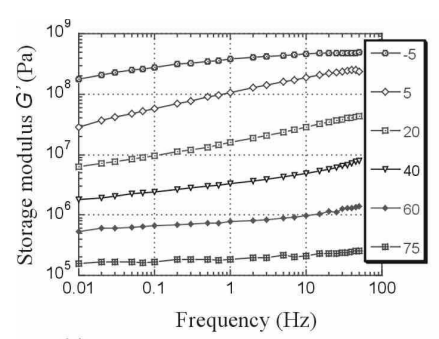

(a)

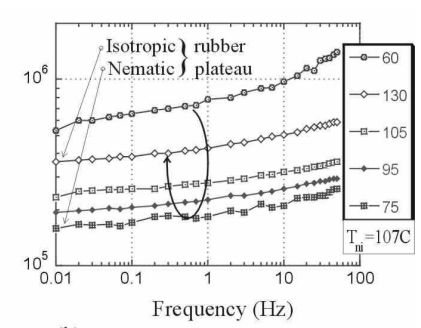

(b)

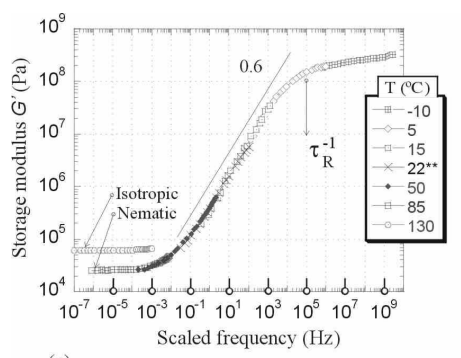

(a)

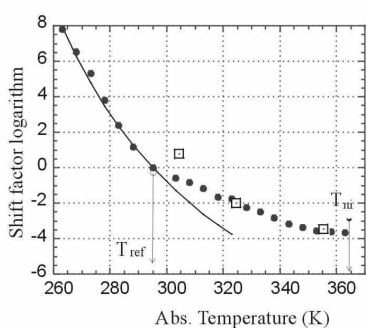

(b)
Fig. 7. Time-temperature superposition of frequency scans for $\mathrm{SiH}$, at different temperatures. (a) The Master Curve built at $T_{\text {ref }}=22^{\circ} C$, superposing the data at temperatures labelled on plot. Annotations also show the expected Rouse time and the power-law exponent of the approach to the glass transition. (b) Logarithm of shift factors plotted against temperature. The curve was fitted with the WLF eq. (11) at lower temperatures. Square symbols refer to the discussion of Section 4 below.
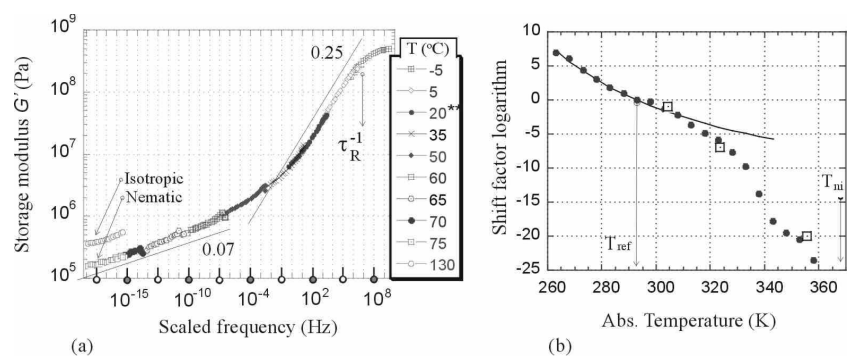

Fig. 8. Time-temperature superposition of frequency scans for SiMC, at different temperatures. (a) The Master Curve built at $T_{\text {ref }}=20^{\circ} \mathrm{C}$, superposing the data at temperatures labelled on plot. Annotations also show the expected Rouse time and the power-law exponents. The plateau level is not reached even at such low extrapolated frequencies. (b) Logarithm of shift factors plotted against temperature. The curve was fitted with the WLF equation at lower temperatures. Square symbols refer to the discussion of Section 4 below.

sults, clearly indicating the effect of dynamic soft elasticity. In addition, one can see for the SiMC material that the low-frequency rubber plateau is not achieved even at highest temperatures: the modulus continues to decrease with decreasing frequency indicating the on-going mechanical relaxation processes in the sheared rubbery network. It is in contrast with the $\mathrm{SiH}$ results, where one can clearly identify the frequency-independent rubber plateau in both the nematic and the isotropic phases.

Below we shall discuss this extremely slow relaxation of the MC-containing elastomers and match these results with the findings of static stress relaxation. We attribute this slowing down to the hairpin folds of the backbone of rods with flexible spacers, which has been demonstrated [25] to freeze the dynamics even of non-crosslinked polymer melts.

\subsection{Time-Temperature Superposition}

The frequency scans presented in Figures 5(a) and 5(a) invite the time-temperature superposition [24]. At the same time, one immediately discovers from the corresponding (b)-plots that such superposition will not be possible as the monodomain nematic rubber sheared in the "soft"
$\boldsymbol{D}$-geometry undergoes the nematic transition. We assert that this conclusion can only be made for materials which show a clean and pronounced soft decrease in $G^{\prime}$ : in a frustrated polydomain elastomer, or in an overcrosslinked network, the required nematic director rotation may be impeded by internal constraints and, therefore, the characteristic nematic soft elasticity masked. In that case, one might expect the ordinary time-temperature superposition to be valid, same as in ordinary isotropic polymer systems.

Time-temperature (or, in this case, frequency-temperature) superposition is a very interesting, practically useful, but also theoretically ill-defined approach to data analysis in dynamic mechanical measurements. The empirical algorithm proposed by Williams, Landel and Ferry (WLF) [24] allows extrapolating the results into the time or frequency ranges far beyond experimentally accessible. As traditional in this field, we attempted such a superposition of our data for nematic elastomers. The storage moduli $G^{\prime}$ taken in the series of frequency scans at different temperatures are shifted along the frequency axis until each data sets superimposes with the previous (with no 
additional vertical shifting). The multiplicative shift factor, $\omega^{\prime}=a_{T} \omega$, is strongly temperature dependent. Another important factor is the choice of reference temperature $T_{\text {ref }}$ at which the real frequency values are taken. WLF suggested using the glass transition temperature as $T_{\text {ref }}$, however, we decided against this for the following reasons. First of all, the presented results show that the dynamic glass transition is, in fact, difficult to pinpoint as the transformation occurs over a range of temperatures. Secondly, no additional information is extracted from the

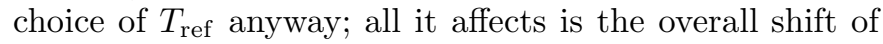
the Master Curve along the frequency axis. Our choice of $T_{\text {ref }}$ as the ambient room temperature was dictated by the possibility of determining the key parameter of polymer chains, the Rouse frequency $\omega_{\mathrm{R}}=\tau_{\mathrm{R}}^{-1}$ at the relevant $T_{\text {ref }}$, from the position of the dynamic glass transition on the frequency axis. In both materials, we find the Rouse time $\tau_{\mathrm{R}} \sim 10^{-5}-10^{-6} \mathrm{~s}$ (which of course must be regarded as a very crude estimate).

Figures 7(a) and 8(a) show the results of superposition for the $\mathrm{SiH}$ and SiMC samples, respectively, in the form of the Master Curves. The procedure is only applicable at the temperatures below the nematic transition $T_{n i}$. Each graph also shows one data set $G^{\prime}(\omega)$, appropriately shifted, at a high temperature, in the isotropic phase. The values are much higher than on the low-frequency nematic plateau and, of course, no shifting along the $\omega$-axis would superimpose them.

The very existence of Master Curves indicates that there is a unique physical process of mechanical relaxation, which spans the range of times, or frequencies that is far beyond experimentally accessible. Our measurements, at each temperature, are only able to detect a small portion of the whole process, but the time-temperature superposition suggests a way of building the whole picture from small pieces. An important part of this analysis is the temperature dependence of shift factors $a_{T}$, which is plotted in Figs. 7(b) and 8(b). The empirical WLF relationship is based on the ideas of glass transition and has been successful in describing the properties of simple homopolymer melts:

$$
\log a_{T}=-\frac{C_{1}\left(T-T_{\mathrm{ref}}\right)}{C_{2}+\left(T-T_{\mathrm{ref}}\right)}
$$

where $C_{1}$ and $C_{2}$ are the constants, called the WLF coefficients. (In more complex polymers, and in crosslinked networks, deviations from this equation have been substantial). In our case, Figures 7 (b) and 8(b), the shift factors follow the WLF relation very well at lower temperatures (below $T_{\text {ref }}$ ), with the WLF coefficients $C_{1} \approx 17.4$ and $C_{2} \approx 102$ for both materials, 1 very close to the values found in ordinary isotropic polymer systems. This is perhaps not surprising when one recalls that the empirical WLF relationship is "designed" to describe the dynamic glass transition region.

However, the shift factors deviate from the classical WLF behavior very strongly as soon as the higher temperatures (and, correspondingly, long relaxation times) are

\footnotetext{
1 Recall that both $\mathrm{SiH}$ and SiMC have been analysed with nearly the same reference temperature, around $20^{\circ} \mathrm{C}$.
}

considered. Here, the two materials behave quite differently: $\mathrm{SiH}$ only allows the superposition of frequency scans with much smaller shifts $a_{T}$, while the Master Curve for SiMC demands much greater shifts. One should note that, in practice, the procedure of shifting of plots along the frequency axis to achieve superposition is very sensitive to the value of each shift factor $a_{T}$ and, accordingly, the errors in Figs. 7(b) and $8(\mathrm{~b})$ are very small. It is more likely that the noise in the $a_{T}$ data is due to uncertainties in DMA temperature or modulus measurements.

Finally, we note that the region of the dynamic glass transition, in both Master Curves for $\mathrm{SiH}$ and SiMC, follows a clear power law $G^{\prime} \propto \omega^{a}$. The plots indicate the non-universal values of the exponent, similar to many previous observations. The very low frequency (long-time) tail of continuing relaxation in SiMC may appear to follow another power law with a much smaller exponent of 0.07 also labelled in Fig. 8(a). However, we are inclined to believe that this is an ambiguity of the complex transient regime: in the previous studies of static stress relaxation [17, 18] we were distinctly unable to distinguish between a very low power-law exponent and an even slower logarithmic relaxation regime. Since the material never reached the equilibrium, whether in a real experiment or in extrapolation, the identification of the longest relaxation regime remained impossible.

\section{Static Stress Relaxation}

Stress relaxation curves were measured at several different temperatures at a constant extensional strain of 0.09 for both $\mathrm{SiH}$ and SiMC, following the simple technique described in [17, 18]. This strain is higher than the shear strain used in DMA experiments (for technical reasons we simply could not impose a smaller extension) and the question of whether the response is still linear may be raised. We have to stress two points. First of all, the strain of $\sim 9 \%$ is, in fact, on the border of the initial linear regime of stress-strain curves measured for both $\mathrm{SiH}$ and SiMC, cf. 233. This, however, has to be regarded with caution because it is impossible to obtain a truly equilibrium stressstrain relation (a certain rate of strain is always involved) and any extrapolation to zero strain rate would involve a theoretical model, which may or may not be ultimately successful. However, as a second point, we have observed that the key conclusion of this section, about the power laws of stress relaxation, remains valid even for higher strains - on and above the soft plateau when we are certain that the stress-strain is non-linear. Therefore, in practice, the choice of the strain step for the static relaxation experiments was not very important.

For the constant-strain extension setup, each sample was cut with a razor blade into a rectangular shape of approximately $6 \times 2.5 \times 0.2 \mathrm{~mm}$, measured by a micrometer (narrow elongated strips minimize the edge effects during the extensional deformation). The initial sample cross-section area of $0.5 \mathrm{~mm}^{2}$ was used to calculate the nominal stress $\sigma$, in units of $\left(\mathrm{mN} / \mathrm{mm}^{2} \equiv \mathrm{kPa}\right)$, from 

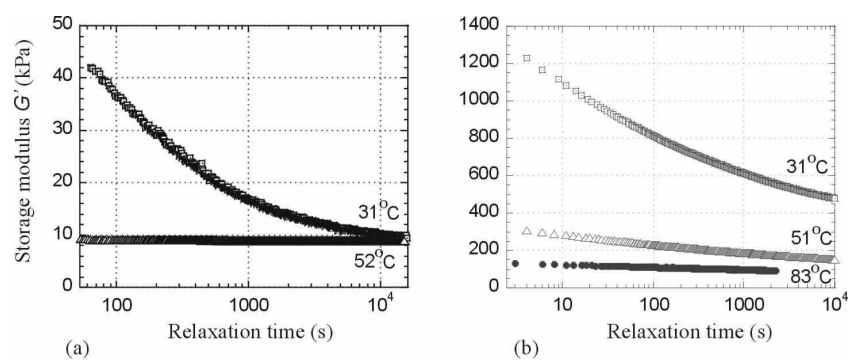

Fig. 9. Typical stress relaxation curves for the $\mathrm{SiH}$ (a) and SiMC (b) samples, after a step-strain of $9 \%$ is applied perpendicular to the nematic director (log-time axis). In each plot, the data sets for different temperatures are labelled.

the force measured by the temperature-compensated dynamometer (UF1/AD20 from Pioden Controls Ltd). The values of force, obtained in arbitrary units, were calibrated with weights at different experimental temperatures of $30,50,80,105^{\circ} \mathrm{C}$. The samples were then extended with the engineering strain $\varepsilon=\left(L-L_{0}\right) / L_{0}$, increasing from 0 to 0.09 within a second. Both monodomain samples, $\mathrm{SiH}$ and SiMC, were stretched perpendicular to their uniform director orientation so that a soft-deformation geometry, comparable to the $\boldsymbol{D}$-shear geometry of DMA experiments, is achieved. In each case the stress relaxation was measured for two days $\left(\sim 10^{5} \mathrm{~s}\right)$. Figure 9 shows typical stress relaxation curves taken in the series of these measurements. The data were then fitted with several possible stress relaxation equations, such as the power-law, the inverse logarithmic law and the exponentials.

In Fig. 9(a), for the "faster" SiH material, one can note that the stress relaxation was substantial at a lower temperature of $31^{\circ} \mathrm{C}$ and is almost unnoticeable at higher temperatures (in fact, the data set for $82^{\circ} \mathrm{C}$ is not shown because it is hard to distinguish from the $52^{\circ} \mathrm{C}$ case). The relaxation in SiMC continues even at high temperatures, but its extent is clearly smaller.

Instead of presenting and discussing the individual relaxation fits and their relations, we shall follow a different route of analysis. If we assume that the Master Curves, shown in Figs. 7(a) and 8(a), are genuine and represent the complete relaxation dynamics in our complex polymeric systems, then we should expect that the same laws must govern the static stress relaxation. Let us plot such a Master Curve against the inverse frequency, which would be a measure of real time. Such plots, Figs. 10(a) and 11.(a) look reasonable, as one would expect the relaxing modulus to behave in real time, in double-logarithmic scale. Of course, one cannot assign anything more than a qualitative meaning to this representation because, let us not forget, the $G^{*}(\omega)$ and $G\left(t-t^{\prime}\right)$ are related by the Fourier transformation and merely mapping $G(\omega)$ onto the inverse frequency is not a rigorous operation. It does, however work for the power-law dependence: from the basic definitions

$$
\begin{aligned}
\sigma(t) & =\int_{-\infty}^{t} G\left(t-t^{\prime}\right) \frac{d}{d t^{\prime}} \varepsilon\left(t^{\prime}\right) d t^{\prime} ; \\
\sigma(\omega) & =\mathrm{i} \omega G^{*}(\omega) \varepsilon(\omega),
\end{aligned}
$$

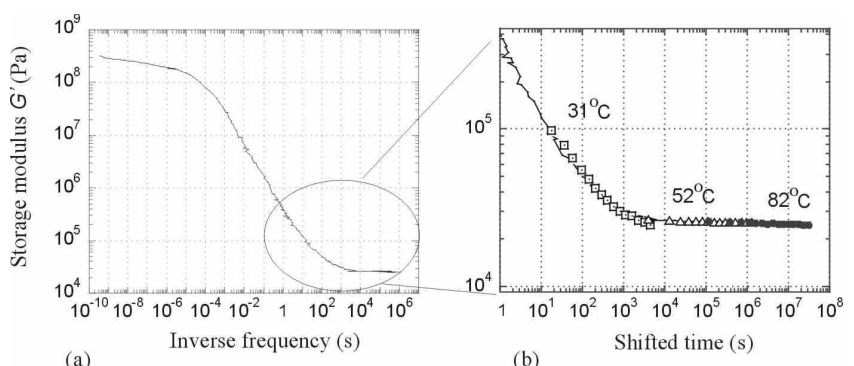

Fig. 10. Stress relaxation analysis for the $\mathrm{SiH}$ material. Graph (a) shows the DMA Master Curve, from Fig. J(a), plotted against the inverse frequency $\omega^{-1}$. The region of long times is expanded in Graph (b), where the real relaxation data from Fig. 9 (a) are shifted along the time-axis to superpose on the same Master Curve. The shift factors required for this superposition are shown as square symbols in Fig. 7(b).
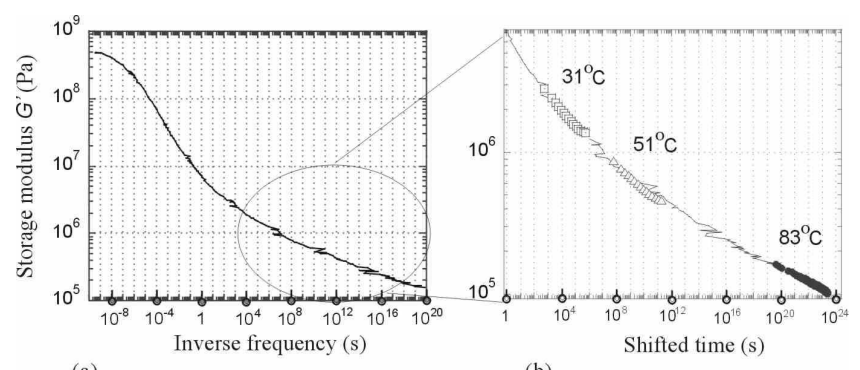

(a)

(b)

Fig. 11. Stress relaxation analysis for the SiMC material. Graph (a) shows the DMA Master Curve, from Fig. B(a), plotted against the inverse frequency $\omega^{-1}$. The region of long times is expanded in Graph (b), where the real relaxation data from Fig. (b) are shifted to superpose on the same Master Curve. The shift factors required for this superposition are shown as square symbols in Fig. \&(b).

and assuming the linear response function to decay as a power law, at long times $G(t) \approx G_{0} t^{-a}$, one can easily calculate the corresponding estimate of the complex shear modulus:

$$
G^{*}(\omega) \approx G_{0} \Gamma(1-a)\left(e^{\frac{1}{2} \mathrm{i} a \pi}-e^{-\frac{3}{2} \mathrm{i} a \pi}\right) \omega^{a} .
$$

Apart from a constant coefficient, this is directly the inverse function of $G(t)$.

Let us now take the real-time stress relaxation data sets and attempt the time-temperature superposition on them, building a Master Curve by shifting along the timeaxis. This operation works for all temperatures, but we only present three data sets in each of Figs. 10(b) and 11(b), to allow a clear view of the underlying line, which is the DMA Master Curve for inverse frequencies. The matching is quite remarkable, especially if we take a look at the shift factors (now for the time-shifts, and so with the opposite sign!) required to bring the relaxation data onto this Master Curve. Figures 7(b) and 8(b) show the frequency shift factors and their WLF analysis, but also the square symbols that represent the (inverse) time shift factors for the static relaxation data. (Recall that the Master Curves were built for the reference temperature of around 
$\left.20^{\circ} \mathrm{C}\right)$. The agreement and the trend in these respective shift factors is not bad.

\section{Discussion}

First of all, we must recognise the difference of our results from some literature data. Gallani et al. and Weilepp et al. [7],9] were both pointing out that the nematic-isotropic transition is trivial for the dynamic mechanical measurements. The reason could be that polydomain nematic elastomers were used, although we have earlier seen the effect of dynamic soft elasticity in a variety of polydomain elastomers [26]. The drop in $G^{\prime}$ below $T_{n i}$ is smaller in polydomain samples, but it only disappears in the monodomain $\boldsymbol{V}$-geometry, as in fact the more recent study $\sqrt{11}$ confirms. It is also interesting that many authors, e.g. [0], 6], reported their success in time-temperature superposition across the nematic-isotropic phase transition, which of course is related to the absence of pronounced soft drops in $G^{\prime}$ values. We can only suppose that a different synthetic procedure of their materials led to the very non-soft (highly semisoft) nematic networks.

Returning to our own results, the two main results of this work are the building of Master Curves and identifying the temperature regimes where the superposition follows the WLF and where it fails altogether, and establishing the direct analogy between the time and the frequency domains. In the first part, the most important observation is the substantial difference in rubber-plateau (low-frequency) moduli between the nematic and isotropic phase, in the shear geometry when the director rotation is induced. This effect of dynamic soft elasticity can lead to a number of technological advances, e.g., in the area of mechanical vibration damping [26] (exploiting the recordhigh values of the loss factor over a broad range of temperatures and frequencies), or even suggesting a novel field of polarised acoustics (using the fact that only certain, soft, shear-wave geometries are strongly attenuated).

Comparing the results of static stress relaxation with those of dynamic mechanical measurements is intriguing. Regarding the ability to quantitatively predict the static relaxation behaviour from the independently (and relatively quickly) acquired DMA data, or vice versa, by inverting the Master Curve at a specific reference temperature, we have to remain cautious. Although the possibility is highly attractive and the procedure has worked spectacularly well in our case, one needs to verify it on different materials, including a check of how the classical isotropic rubber behaves in this respect.

In our case, the Master Curve inversion has been successful and predicts that, for instance, a "traditional" 19 , 20 siloxane side-chain nematic elastomer $\mathrm{SiH}$, crosslinked in a way that allows the soft elasticity, would be able to relax under a constant strain and reach its mechanical equilibrium (at least approximately) after some $10^{4} \mathrm{~s}$ at room temperature, with a very little residual relaxation after that. This is somewhat different from the results [17] on a very similar, but polydomain nematic elastomer, where the relaxation have not reached its end even after extrapolation to astronomical times. Analogous continuing relaxation was reported in other, chemically very different polydomain nematic rubbers 18. The effect of internal mechanical constraints imposed by the domain interfaces is clearly dominating long-time regime, but is absent in our present monodomain network. In contrast, the composite nematic elastomer SiMC, albeit monodomain, also shows a non-ending relaxation. We assume, by analogy with the previous work on relaxation in non-crosslinked MC polymer [25], that internal constraints on "free" stress relaxation are now imposed by the hairpin folds of long nematic main-chain polymer strands.

In summary - dynamic-mechanical analysis have been performed using two different monodomain nematic elastomers having different degrees of anisotropy and internal microstructure. Master Curves have been built between the glassy state and the nematic-isotropic phase transition, where the modulus saturates on the low-level soft plateau. Above $T_{n i}$ the modulus rises substantially, since the internal relaxation is no longer able to reduce the elastic response - and the further time-temperature superposition fails. The dynamics of these elastomers is dominated by power laws (non-universal, depending on the material and temperature), which is confirmed by the successful operation of the Master Curve inversion to describe the static stress relaxation. Power laws (in time) of stress relaxation match very well with the corresponding frequency dependence of the dynamic modulus in the appropriate range of temperatures.

\section{Acknowledgements}

We thanks EPSRC and Bridgestone Corporation for funding this work, and Wacker Chemie for the donation of the platinum catalyst. We are indebted to H. Finkelmann for the advice and assistance in chemical synthesis and to A.R. Tajbakhsh for the preparation of monodomain LCE materials.

\section{References}

1. H. Finkelmann, H.J. Koch and G. Rehage G Macromol. Rapid Comm. 1981, 2, 317.

2. K.F. Wissbrun J. Rheol. 1981, 25, 619.

3. K.H. Hanus, W. Pechhold, F. Soergel, B. Stoll and R. Zentel Colloid Polym. Sci. 1990, 268, 222.

4. T. Pakula Makromol. Chem. 1991, 192, 2401.

5. R.H. Colby, J.R. Gillmor, G. Galli, M. Laus, C.K. Ober and E. Hall Liquid Crystals 1993, 13, 233.

6. V. Fourmaux-Demange, A. Brulet, J.P. Cotton, L. Hilliou, P. Martinoty, P. Keller and F. Boue Macromolecules 1998, 31, 7445 .

7. J.L. Gallani, L. Hilliou and P. Martinoty Phys. Rev. Lett. 1994, 72, 2109.

8. J.L. Gallani, L. Hilliou, P. Martinoty, F. Doublet and M. Mauzac J. Physique II 1996, 6, 443.

9. J. Weilepp, P. Stein, N. Assfalg and H. Finkelmann Euro. Phys. J. E 1999,47, 508 
10. M. Doi and S.F. Edwards, Theory of Polymer Dynamics 1986, Clarendon Press, Oxford.

11. P. Stein, N. Assfalg, H. Finkelmann and P. Martinoty, Euro. Phys. J. E 2001, 4, 255.

12. S.M. Clarke, A.R. Tajbakhsh, E.M. Terentjev and M. Warner Phys. Rev. Lett. 2001, 86, 4044.

13. S.M. Clarke, A. Hotta, A.R. Tajbakhsh and E.M. Terentjev Phys. Rev. E 2002, 65, 021804.

14. E.M. Terentjev and M. Warner Eur. Phys. J. E 2001, 4, 343.

15. R. Chasset and P. Thirion, P.; In: Proceedings of the Conference on Physics of Non-Crystalline Solids; J. A. Prins, Ed.; North-Holland Publishing Co.: Amsterdam, 1965; p. 345.

16. J.G. Curro and P. Pincus Macromolecules 1983, 16, 559.

17. S.M. Clarke and E.M. Terentjev Phys. Rev. Lett. 1998, $81,4436$.

18. A. Hotta and E.M. Terentjev J. Phys. C: Cond. Mat. 2001, 13, 11453.

19. J. Küpfer and H. Finkelmann, Macromol. Chem. Rapid. Comm. 1991, 12, 717.

20. J. Küpfer and H. Finkelmann Makromol. Chem. Phys. 1994, 195, 1353.

21. A.R. Tajbakhsh and E.M. Terentjev Euro. Phys. J. E 2001, 6, 181.

22. V. Percec and M. Kawasumi Macromolecules 1991, 24, 6318.

23. S.M. Clarke, A. Hotta, A.R. Tajbakhsh and E.M. Terentjev Phys. Rev. E 2001, 64, 061702.

24. J.D. Ferry Viscoelastic Properties of Polymers; John Wiley \& sons: New York, 1980.

25. F. Elias, S.M. Clarke, R. Peck and E.M. Terentjev Europhys. Lett. 1999, 47, 442.

26. S.M. Clarke, A.R. Tajbakhsh, E.M. Terentjev, C. Remillat, G.R. Tomlinson and J.R. House J. Appl. Phys. 2001, 89, 6530 . 